\title{
BABIES BORN BETTER: O USO DO SOFTWARE MAXQDA NA ANÁLISE PRELIMINAR DAS RESPOSTAS PORTUGUESAS À SECÇÃO QUALITATIVA DO INQUÉRITO
}

\author{
BABIES BORN BETTER: THE USE OF MAXQDA IN A PRELIMINARY \\ ANALYSIS OF PORTUGUESE RESPONSES TO THE QUALITATIVE \\ SECTION OF THE SURVEY
}

\author{
Dulce Morgado Neves ${ }^{1}$ \\ Mário JDS Santos ${ }^{2}$
}

\begin{abstract}
Resumo: A saúde materna envolve um vasto conjunto de conhecimentos e práticas que visam não só a promoção da saúde na gravidez e parto mas também uma experiência que seja entendida como positiva pelas mulheres. O inquérito Babies Born Better tem como objetivo informar o debate público sobre a qualidade dos cuidados de saúde, partindo do discurso das mulheres. Baseado na componente qualitativa do inquérito, este artigo consiste num ensaio de recurso ao MaxQDA para a análise das respostas portuguesas. A utilização deste software revelou ser útil à mediação entre os dados e a análise, facilitando a sistematização da informação. A análise veio salientar a importância da relação entre as mulheres e os profissionais de saúde, enquanto fator estruturante das experiências de parto.
\end{abstract}

Palavras-chave: Babies Born Better; MaxQDA; Saúde Materna; Portugal.

Abstract: Maternal health involves a wide range of knowledge and practices whose purpose is to promote health in pregnancy and birth and an experience understood as positive by women. The survey Babies Born Better was designed to inform the public debate about the quality of care, learning from women's discourses. Based on the qualitative component of the survey, this paper is an exercise in the use of MaxQDA in the analysis of Portuguese responses. The use of this software proved to be useful to the mediation between data and the analysis, allowing the systematization data. The analysis comes to reveal the importance of the relationship between women and health professionals, as a structuring factor of childbirth experiences.

Keywords: Babies Born Better; MaxQDA; Maternal health; Portugal.

\section{Introdução: a investigação em saúde materna}

Uma grande parte do conhecimento produzido sobre a saúde materna e as condições em que ocorre o parto baseia-se em metodologias quantitativas e análises extensivas, com o recurso a informação estatística, a indicadores objetivos e a dados demográficos, reflectindo o atual predomínio de um paradigma positivista na saúde e

\footnotetext{
${ }^{1}$ Doutorada em Sociologia, Instituto Universitário de Lisboa (ISCTE-IUL). Instituto Universitário de Lisboa (ISCTE-IUL), CIES-IUL, Lisboa, Portugal. Email: dulce neves@iscte-iul.pt

${ }^{2}$ Mestre em Saúde, Medicina e Sociedade, Instituto Universitário de Lisboa (ISCTE-IUL). Instituto Universitário de Lisboa (ISCTE-IUL), CIES-IUL, Lisboa, Portugal. Email: mjdss@iscte-iul.pt
} 
deixando para segundo plano as contribuições das abordagens qualitativas (CLAUSEN; SANTOS, 2017; PINTASSILGO, 2016).

O enfoque na diminuição das taxas de mortalidade materna e perinatal e a relevância dos cálculos do risco em saúde materna têm legitimado o aumento da vigilância e da intervenção obstétrica, sob um modelo de atenção ao nascimento fortemente institucionalizado e medicalizado (CONRAD, 2007; PINTASSILGO, 2016). Com efeito, muitos dos serviços de saúde materna privilegiam, em grande medida, a gestão do risco, subvalorizando a importância de se promover uma experiência positiva na gravidez, parto e pós-parto.

No entanto, o nascimento é um fenómeno complexo, que integra dimensões de ordem social, cultural, política e económica, fazendo das abordagens transdisciplinares particularmente adequadas à compreensão dos programas de saúde materna e à melhoria dos cuidados prestados.

Naturalmente que a emergência de abordagens mais inclusivas não é alheia ao movimento pela humanização do nascimento ou à defesa de um cuidado holístico, respeitador da agência, singularidade e integridade de cada pessoa (VAN TEIJLINGEN; FRITH; CHURCH, 2017). Neste sentido, se é verdade que no que à saúde materna diz respeito continua a haver uma lacuna no conhecimento a respeito da perceção individual e subjetiva da mulher sobre a sua própria experiência de gravidez e parto, também é certo que novas perspetivas vão ganhando lugar e denunciando as insuficiências das avaliações baseadas apenas em indicadores objetivos, como as taxas de mortalidade materna, ou na prevalência de determinadas intervenções obstétricas.

Ilustrativas da emergência de um novo paradigma também ao nível político e institucional, as Recomendações da OMS sobre cuidados pré-natais para uma experiência positiva na gravidez, vêm reforçar

\begin{abstract}
a importância de estabelecer uma comunicação efetiva com as mulheres grávidas acerca de questões fisiológicas, biomédicas, comportamentais e socioculturais, e de um apoio respeitoso e efetivo, incluindo seus aspetos sociais, culturais, emocionais e psicológicos. Estas funções de comunicação e apoio aos cuidados pré-natais são essenciais, não só para salvar vidas, mas também para melhorar a vida, a utilização dos cuidados de saúde e a sua qualidade. As experiências positivas das mulheres durante os cuidados prénatais e o parto podem constituir a base de uma maternidade saudável (ORGANIZAÇÃO MUNDIAL DA SAÚDE, 2016, p. 1).
\end{abstract}

Partindo da análise preliminar de uma secção de questões de natureza qualitativa, mas integrantes de um inquérito internacional extensivo, o presente artigo vem então reforçar a importância da complementaridade entre métodos e técnicas de recolha e 
análise empírica, ao mesmo tempo que se insere numa matriz de pensamento que valoriza as dimensões subjetivas das experiências de gravidez e parto enquanto aspetos centrais para o conhecimento e melhoria da qualidade dos cuidados de saúde materna. Os resultados desta análise foram apresentados pela primeira vez no $6^{\circ}$ Congresso IberoAmericano em Investigação Qualitativa (NEVES; SANTOS, 2017), tendo agora merecido uma revisão.

\section{Babies Born Better: contexto e objetivos}

Neste artigo pretendemos apresentar uma análise exploratória e preliminar das respostas de mulheres portuguesas à secção qualitativa de um inquérito internacional intitulado Babies Born Better.

Babies Born Better é um projeto que teve origem na COST Action IS0907 "Childbirth Cultures, Concerns and Consequences: Creating a dynamic EU framework for optimal maternity care" e que foi criado com o objetivo de conhecer a opinião e as experiências de mulheres, de diferentes países, em termos da assistência recebida durante o parto e o trabalho de parto.

Desde o seu lançamento em 2012 em inglês, o inquérito foi traduzido em diversas línguas e lançado online em mais de 40 países, obtendo no total cerca de 40.000 respostas de mulheres que contavam com uma experiência de parto há menos de 5 anos. Depois de uma primeira fase (2012-2015), o inquérito foi sujeito a revisões e foi relançado recentemente com um novo formato. Em Portugal, entre abril de 2014 e setembro de 2015, foram recolhidas 1678 respostas e será sobre estas que a presente análise irá incidir.

\section{Estratégia Metodológica}

\subsection{A dimensão qualitativa do inquérito Babies Born Better}

Todo o processo de conhecimento da realidade social requer procedimentos de análise de dados de elevada complexidade. Aprender a partir do discurso das mulheres sobre as suas experiências de parto a fim de informar o debate público e político sobre a qualidade da assistência em saúde materna era uma das finalidades do inquérito Babies Born Better. Nesse sentido, para além de se propor a obter uma caracterização global da gravidez e do parto das mulheres por meio de categorias previamente definidas, o 
inquérito compreendeu também uma secção de questões às quais as inquiridas deveriam responder abertamente e por palavras suas sobre as características das suas experiências de parto, nomeadamente no que se refere a aspetos que consideraram positivos e negativos dessas vivências tão singulares.

É sobre esta componente qualitativa (complementar e enriquecedora do pendor eminentemente quantitativo e extensivo da pesquisa) que iremos focar a nossa atenção, propondo-nos a conhecer em maior profundidade os sentidos atribuídos pelas próprias mulheres às suas experiências.

Concretamente, serão as respostas obtidas às seguintes questões do inquérito aquelas sobre as quais nos iremos debruçar nesta abordagem:

- Quais foram as três melhores coisas nos cuidados que recebeu? Por favor coloque a melhor no topo da lista.

- Se tivesse o poder para fazer três mudanças nos cuidados que teve, quais seriam essas mudanças? Por favor coloque a mudança mais importante no topo da lista.

- Imagine que uma amiga muito próxima ou uma familiar está grávida. Pediramlhe para fazer uma descrição verdadeiramente honesta dos cuidados que recebeu no local onde teve o seu último bebé. Pode usar até seis palavras ou frases. Quais serão?

- Por favor escreva aqui quaisquer comentários que queira fazer. Poderá explicar as suas respostas com mais detalhe, ou adicionar qualquer outra informação que gostaria que soubéssemos sobre a sua experiência com os cuidados de saúde materna.

\subsection{O recurso ao MaxQDA}

Numa primeira etapa de análise dos dados de natureza qualitativa provenientes da aplicação do inquérito Babies Born Better, têm vindo a ser exploradas as potencialidades de um software específico de CAQDAS, o MaxQDA (MaxQDA, 2017). CAQDAS é o acrónimo de Computer-Aided Qualitative Data Analysis Software, ou seja, um termo genérico para definir programas de análise de dados qualitativos assistida por computador.

Softwares como o MaxQDA foram desenvolvidos na esteira das aspirações metodológicas associadas à grounded theory, com o intuito de facilitar e enriquecer um conjunto de tarefas relacionada com análise de conteúdo. Como veremos, aliás, o recurso a tal programa pode servir de mediação entre os dados empíricos e a análise desenvolvida pelos investigadores, servindo todas as fases do processo de análise qualitativa, tal como 
ele é descrito por Miles, Huberman e Saldaña (2013), desde a codificação dos dados, a sua transformação e até à obtenção de resultados.

Tendo resultado de um inquérito online, as respostas à versão portuguesa do inquérito Babies Born Better foram disponibilizadas pelo Comité de Gestão do inquérito à equipa de colaboradores portugueses do projeto sob a forma de um documento Excel estruturado, onde cada linha correspondia a uma mulher inquirida e cada coluna a uma resposta. Uma vez que os dados recolhidos que nos propusemos analisar eram muito vastos, a estratégia metodológica passou, em primeiro lugar pela importação e codificação automática dos parágrafos por questão. Note-se que, neste processo, foram também importadas variáveis de caracterização dos sujeitos (como a idade, país de residência, língua, cidade mais próxima, quantos filhos deu à luz, ano do último parto, local do parto, e grupo de cuidadores principais) para melhor contextualizar as respostas abertas.

Foi necessário proceder, depois, a uma verificação e limpeza da codificação automática dos documentos, já que algumas das inquiridas não tinham respondido às questões abertas e havia respostas referentes a partos no estrangeiro. Dos 1678 inquéritos iniciais foram, finalmente, consideradas 1348 respostas.

Procedeu-se à personalização e organização dos códigos e das variáveis atribuídas automaticamente. O nome automático das variáveis e dos códigos foi também traduzido e adaptado para português. Os códigos foram organizados em categorias e subcategorias. Algumas variáveis foram definidas como tooltips - etiquetas que permitem o acesso rápido a alguns dados de caracterização quando se seleciona um documento ou quando são devolvidos excertos. Para facilitar a sua identificação e para mais facilmente se distinguirem dos códigos atribuídos automaticamente com os códigos provenientes da análise, a primeira foi feita com emoticodes, e os códigos e subcódigos resultantes da análise foram identificados com um nome e uma cor.

Uma vez terminado este tratamento inicial, iniciou-se a análise propriamente dita. Foi feita uma contagem simples e exploratória das palavras (word count), de forma a fazer um levantamento das palavras mais frequentes. Aí, foram ensaiadas várias pesquisas com diferentes palavras-chave para testar a sua adequação. Dos resultados obtidos, foi construída uma lista com as palavras ou grupos de palavras mais relevantes e mais frequentes. Estas foram organizadas em três categorias:

- Características dos cuidados recebidos

- Intervenções e procedimentos obstétricos 
- Grupos profissionais

Fez-se depois uma lexical search para cada uma das palavras dentro das categorias referidas. Recorreu-se à ferramenta "Autocode/Export: ignore hit" sempre que necessário, para excluir palavras ou sentidos diferentes do que se procurava. Optou-se por codificar sempre o parágrafo e não apenas a palavra, para melhor apreensão do contexto.

\section{Alguns resultados da análise}

O processo de codificação e a análise exploratória feitos até ao momento permitem destacar um conjunto relevante de informação sobre as experiências de parto em Portugal.

Antes de mais, algumas considerações sobre a amostra: é composta por 1348 mulheres que tiveram pelo menos um filho há menos de 5 anos, em Portugal. A idade varia entre 18 e 48 anos, com uma idade média de 35 anos e a modal de 33 anos.

Os partos ocorreram maioritariamente no hospital (94,9\%), mas um número considerável de mulheres referiu ter tido um parto em casa (4,5\%). Embora com limitações, os dados do Instituto Nacional de Estatística português indicam cerca de 1\% de partos em casa apenas, pelo que existe uma clara sobre-representação de partos em casa na nossa amostra.

Os locais de nascimento distribuem-se por todo o país. A maioria dos partos (83\%) foi de termo - entre as 37 e as 42 semanas - e a maior parte das mulheres $(67,5 \%)$ referiu não ter tido problemas durante a gravidez. Aproximadamente metade das mulheres disse ter sido cuidada por uma combinação de médicos/as e enfermeiros/as ou parteiros/as. Apenas 16,4\% refere terem sido os/as médicos/as os/as principais cuidadores/as. De destacar que os/as enfermeiros/as constituem o grupo profissional mais representado isoladamente.

Quando nos centramos nas respostas à secção de apreciação qualitativa das experiências de parto, verificamos que as descrições são muito diversificadas. Numa primeira análise, e tendo em conta apenas o número médio de respostas, nota-se que foram mencionados mais aspetos positivos que negativos na descrição dos cuidados recebidos (tabela 1 ).

Tabela 1: Média de respostas a cada secção do inquérito

\begin{tabular}{cccc}
\hline Secção do inquérito & Número de questões & Média de respostas & $\mathbf{n}$ \\
\hline Melhores coisas & 3 & 2,7 & 3590 \\
O que mudaria & 3 & 2,0 & 2698
\end{tabular}




\begin{tabular}{cccc} 
Descrição da experiência & 6 & 4,0 & 5439 \\
Comentários & 1 & 0,4 & 603 \\
\hline Total & $\mathbf{1 3}$ & $\mathbf{9 , 1}$ & $\mathbf{1 2 3 3 0}$
\end{tabular}

Fonte: Cálculos próprios a partir da base de dados portuguesa do Babies Born Better (2014-2015)

\subsection{Características dos cuidados recebidos}

A análise das palavras usadas para descrever os cuidados recebidos permite obter um retrato aproximado da experiência na amostra (figura 1). A atenção, o apoio, a simpatia, o profissionalismo e o respeito são, como se vê, as características mais frequentemente mencionadas no total das secções analisadas.

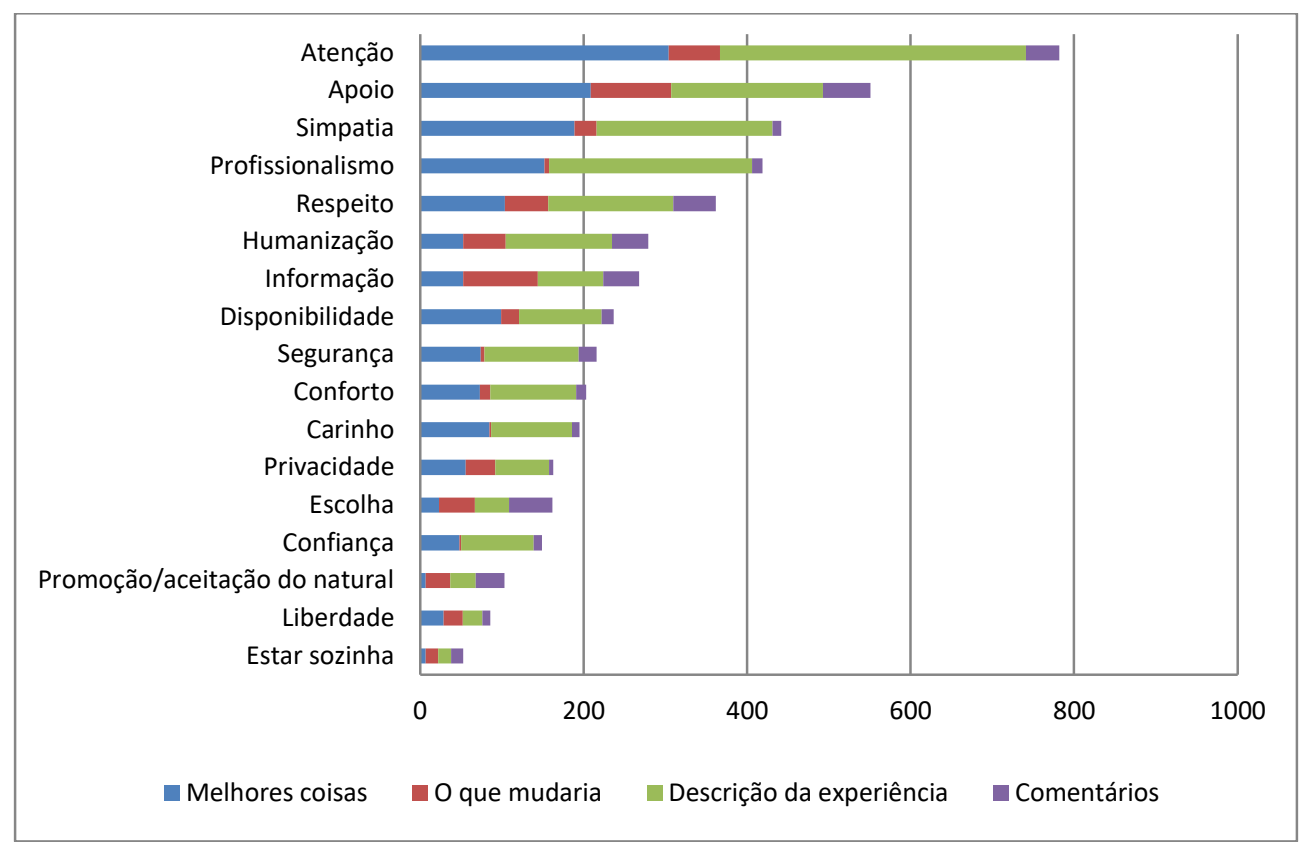

Figura 1: Características dos cuidados recebidos por secção do inquérito

Fonte: Cálculos próprios a partir da base de dados Portuguesa do Babies Born Better (2014-2015)

Quando limitamos a análise às duas primeiras secções - "as melhores coisas" e "o que mudaria" - é possível analisar com maior detalhe se uma dada característica foi mencionada como aspeto positivo ou negativo da experiência (figura 2). 


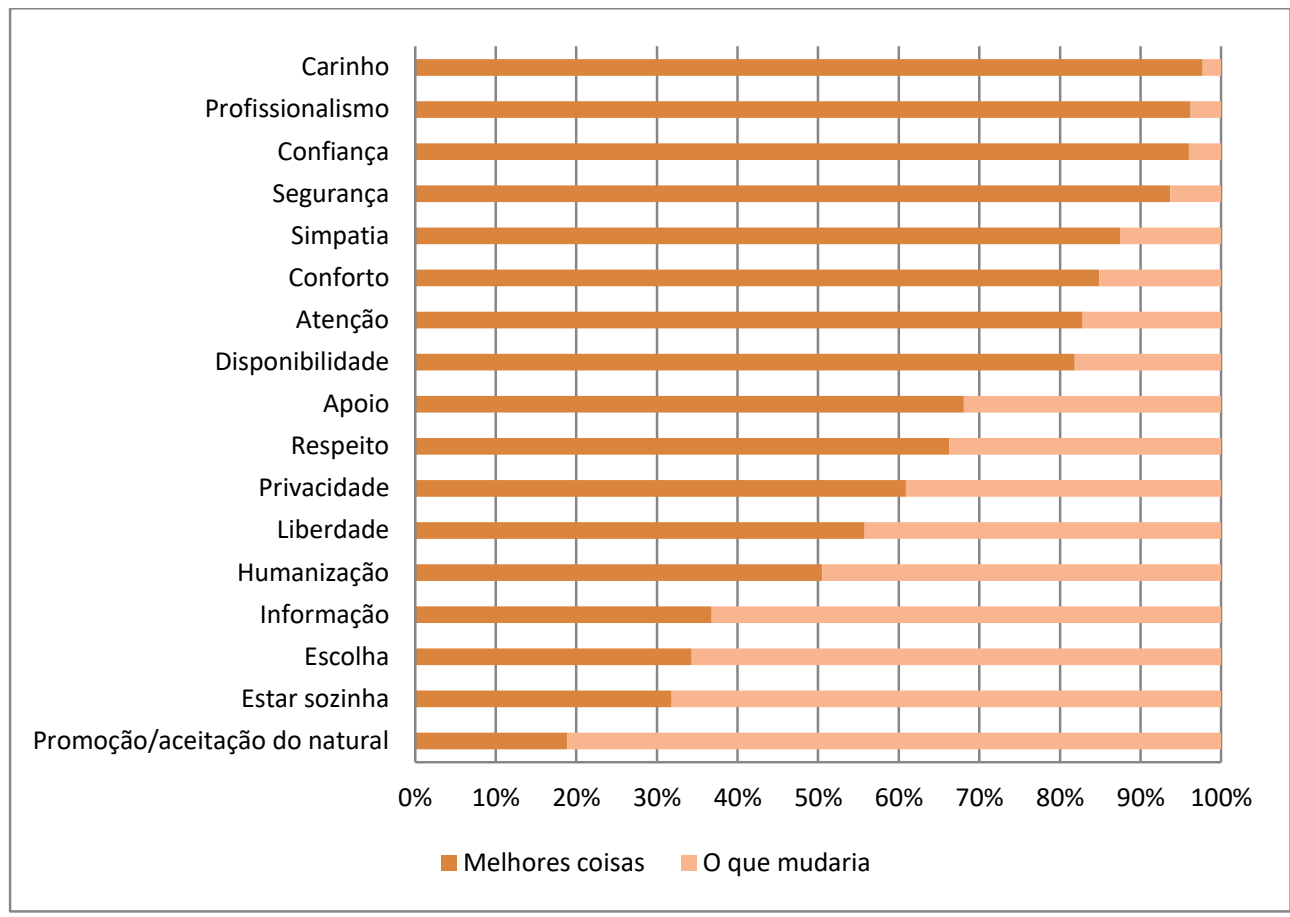

Figura 2: Características dos cuidados recebidos como aspetos positivos ou a melhorar Fonte: Cálculos próprios a partir da base de dados Portuguesa do Babies Born Better (2014-2015)

Desta análise, verificamos que os aspetos que dizem respeito à dimensão interrelacional (nomeadamente à relação estabelecida com os atores profissionais ou à sua atitude) são mais vezes referidos como aspetos positivos. Pelo contrário, as características associadas à agência dos sujeitos (as mulheres) ou à sua condição na experiência dos cuidados são mais frequentemente mencionadas enquanto questões a melhorar.

\subsection{Intervenções e Procedimentos Obstétricos}

No que respeita a intervenções obstétricas é de notar que a sua referência surge mais vezes associada a aspetos considerados negativos da experiência de parto (figura 3 ).

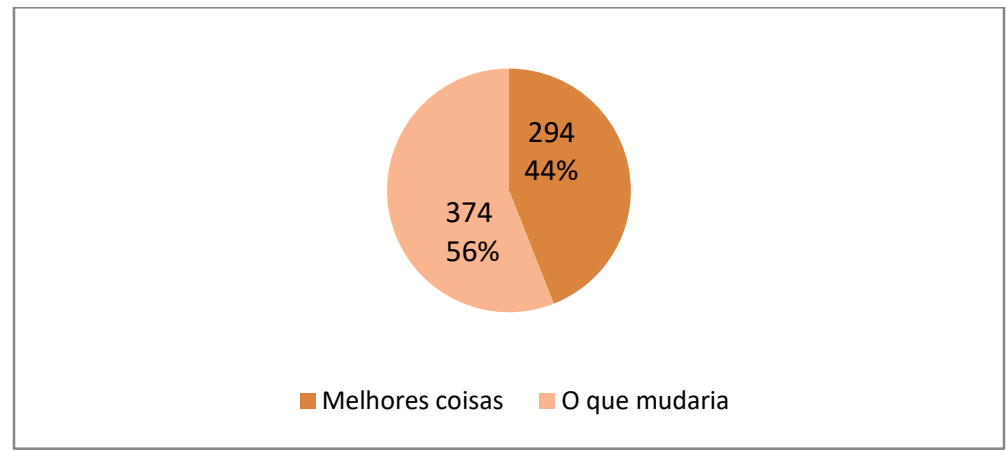

Figura 3: Total de referências a intervenções e procedimentos obstétricos como aspetos positivos ou a melhorar

Fonte: Cálculos próprios a partir da base de dados Portuguesa do Babies Born Better (2014-2015) 
Analisando os resultados em maior detalhe, verificamos que a analgesia administrada para alívio da dor, o recurso ao banho ou água ou à bola de pilates durante o trabalho de parto (métodos não farmacológicos de alívio da dor), ou a possibilidade de fazer "pele com pele" com o bebé (para além de referências mais genéricas a "tratamento" ou "vigilância") são as únicas intervenções mais vezes referidas como aspetos positivos das experiências. As demais intervenções mencionadas surgem nos discursos predominantemente associadas aos aspetos negativos, o que, independentemente da legitimidade ou da pertinência do recurso a cada uma das intervenções e técnicas referidas, pode remeter para as representações associadas à cultura intervencionista que caracteriza os cuidados de saúde materna na contemporaneidade (figura 4).

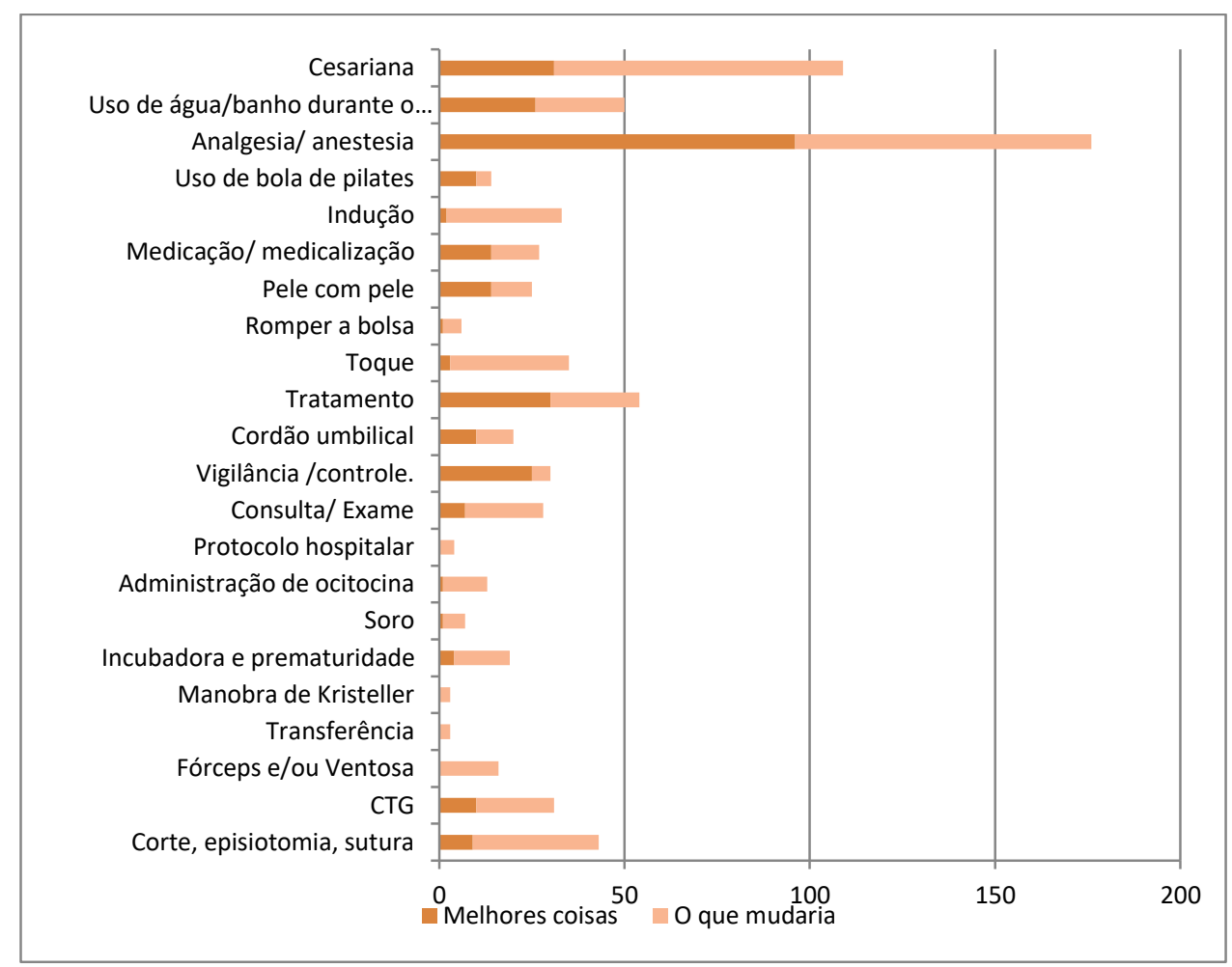

Figura 4: Intervenções obstétricas associadas a aspetos positivos ou a melhorar Fonte: Cálculos próprios a partir da base de dados Portuguesa do Babies Born Better (2014-2015)

\subsection{Grupos Profissionais}

As categorias profissionais que intervêm nos serviços de saúde materna e na assistência ao parto em particular constituem outra das categorias importantes de caracterização das experiências de nascimento. Nos discursos analisados sobre aspetos positivos ou negativos são os enfermeiros o grupo profissional mais frequentemente mencionado, com 299 referências nas apreciações positivas e 173 nos aspetos a melhorar. 
A seguir aos enfermeiros, os médicos foram também referidos em muitas respostas, sendo mais vezes mencionados para reportar aspetos positivos das experiências. Em menor número, doulas e auxiliares constituem outros grupos profissionais mencionados (figura 5). A referência a doulas merece aqui uma especial atenção porque embora, em termos estritamente contabilísticos, a sua menção por parte das inquiridas seja mais frequente nos aspetos a melhorar, uma análise mais atenta dos dados vem revelar-nos que dos 9 excertos que colocavam as doulas no âmbito das apreciações negativas da experiência, apenas uma dizia efetivamente respeito a uma crítica à atuação da doula, sendo as restantes 8 referências críticas às limitações impostas pelas instituições hospitalares à presença e atuação da doula, tal como ilustra o seguinte excerto: "[Gostaria de poder ter tido] a presença de uma doula sem que o meu marido tivesse que sair [da sala de partos]."

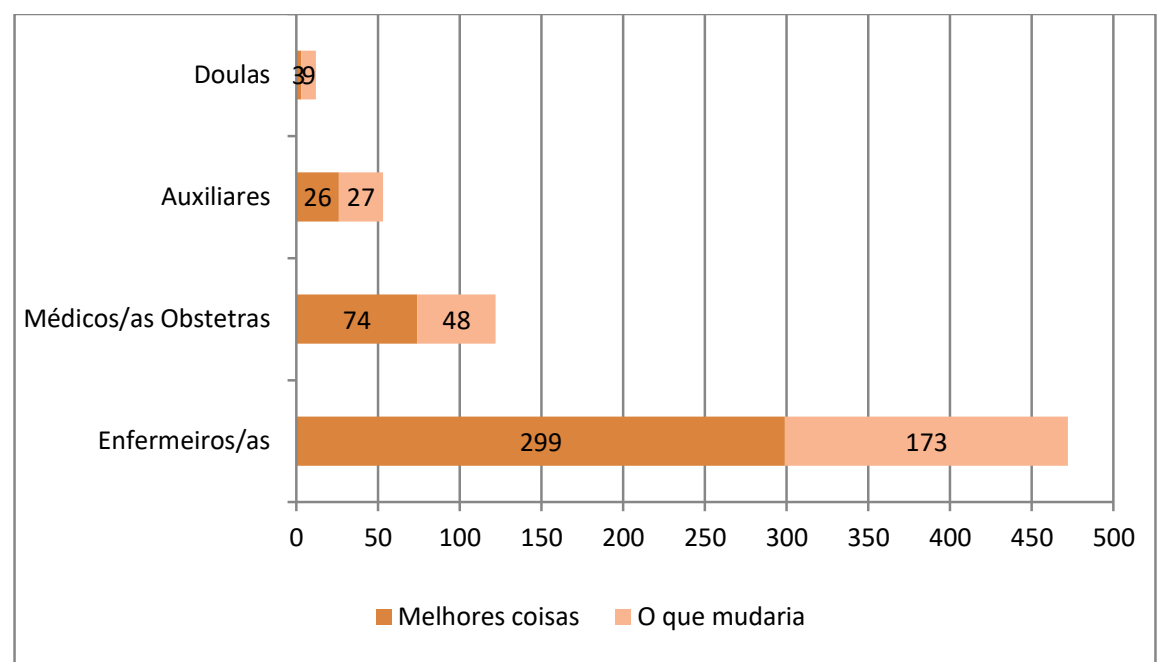

Figura 5: Grupos profissionais associados a aspetos positivos ou a melhorar

Fonte: Cálculos próprios a partir da base de dados Portuguesa do Babies Born Better (2014-2015)

\section{Conclusões}

Ao mesmo tempo que procurou ser um subsídio para a valorização das dimensões subjetivas das experiências de gravidez e parto das mulheres, este artigo veio também reforçar a importância da complementaridade entre métodos e técnicas de análise empírica para o estudo de fenómenos multidimensionais como o nascimento. Em particular, partindo da análise preliminar de uma secção de questões de natureza qualitativa, mas integrantes de um inquérito internacional extensivo, quisemos ensaiar o recurso a um software de análise qualitativa de dados assistida por computador (CAQDAS) e beneficiar das suas funcionalidades para a análise dos discursos recolhidos. 
A utilização do MaxQDA revelou ser útil e adequada para a mediação entre os dados recolhidos e a análise construída. Num contexto em que as respostas provêm da aplicação de um inquérito por questionário com a dimensão do Babies Born Better, o recurso a este software revela-se particularmente vantajoso na funcionalidade de sistematização da informação que, sendo de natureza qualitativa, foi recolhida de forma extensiva. Aqui, apenas foram descritos os elementos de uma primeira fase do processo de análise, sendo agora objetivo aprofundar a análise com uma categorização mais exaustiva das respostas, uma verificação mais pormenorizada dos excertos codificados e, claro, uma exploração mais aprofundada das potencialidades da utilização do MaxQDA para a obtenção de resultados.

Ainda assim, desta primeira análise dos dados das secções qualitativas do inquérito Babies Born Better foi já possível constatar a relevância da relação estabelecida entre as mulheres e os profissionais que as acompanham e assistem no momento do parto, sendo esse fator estruturante da configuração de outros aspetos mencionados (positiva ou negativamente) pelas mulheres.

\section{Referências}

CLAUSEN, J. A.; SANTOS, MJD Capturing the complexity of practice as an insider: in-labour ethnography. In: CHURCH, S. et al. (Orgs.). New thinking on improving maternity care international perspectives. 1. ed. London: Pinter and Martin, 2017. p. 73-89.

CONRAD, P. The medicalization of society. 1. ed. Baltimor: The Johns Hopkins University Press, 2007.

MAXQDA. Software for qualitative data analysis. Version 12. Berlin: VERBI Software Consult - Sozialforschung GmbH. Disponível em: 〈http://www.maxqda.com/〉. Acesso em: 03 nov. 2016. 2017.

MILES, M.; HUBERMAN, A. M.; SALDAÑA, J. Qualitative Data Analysis - A Methods Sourcebook. 3. ed. Thousand Oaks: Sage, 2013.

NEVES, D. M.; SANTOS, MJD. A componente qualitativa do inquérito Babies Born Better: Análise preliminar às respostas portuguesas com recurso ao MaxQDA. In: CONGRESSO IBERO-AMERICANO EM INVESTIGAÇÃO QUALITATIVA, 6, 2017, Salamanca. Anais... Salamanca: Universidade Lusófona do Porto, 2017. p. 422-430. Disponível em: <http://proceedings.ciaiq.org/index.php/ciaiq2017/article/view/1184/1146> Acesso em: 11 jul. 2017.

ORGANIZAÇÃO MUNDIAL DA SAÚDE. Recomendações da OMS sobre cuidados prénatais para uma experiência positiva na gravidez. Geneva: WHO. Disponível em:

〈http://apps. who.int/iris/bitstream/10665/250800/2/WHO-RHR-16.12-por.pdf〉. Acesso em: 10 Ago. 2017. 2016. 
PINTASSILGO, S. C. Condições do nascimento e indicadores de saúde materna em Portugal. In: CUNHA, V. et al. (Orgs). A(s) problemática(s) da natalidade em Portugal. Uma questão social, económica e política. 1. ed. Lisboa: Imprensa de Ciências Sociais, 2016. p. 153-159.

VAN TEIJLINGEN, E.; FRITH, L.; CHURCH, S. Introduction: Maternity care international perspectives: setting the scene. In: CHURCH, S. et al. (Orgs.). New thinking on improving maternity care - international perspectives. 1. ed. London: Pinter \& Martin, 2017. p. 9-18.

Recebido em: 24 de março de 2018.

Aceito em: 04 de abril de 2018. 